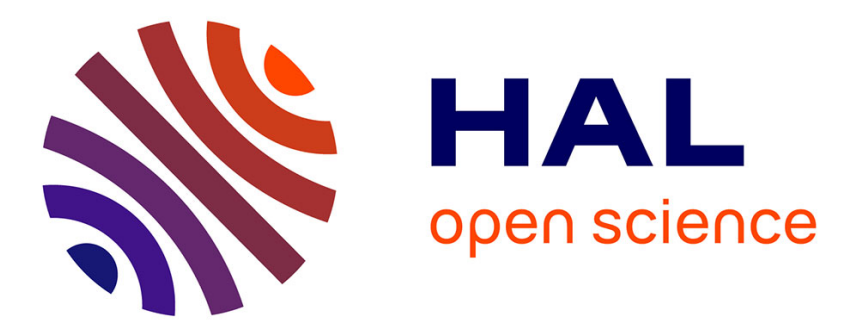

\title{
Droit de propriété et réforme du secteur d'État Thierry Pairault
}

\section{- To cite this version:}

Thierry Pairault. Droit de propriété et réforme du secteur d'État. Études Chinoises, 2001, 20 (1-2), pp.3-37. halshs-00076579

\section{HAL Id: halshs-00076579 \\ https://shs.hal.science/halshs-00076579}

Submitted on 25 May 2006

HAL is a multi-disciplinary open access archive for the deposit and dissemination of scientific research documents, whether they are published or not. The documents may come from teaching and research institutions in France or abroad, or from public or private research centers.
L'archive ouverte pluridisciplinaire HAL, est destinée au dépôt et à la diffusion de documents scientifiques de niveau recherche, publiés ou non, émanant des établissements d'enseignement et de recherche français ou étrangers, des laboratoires publics ou privés. 


\title{
Droit de propriété et réforme du secteur d'État
}

\author{
Thierry Pairault ${ }^{1}$
}

La réforme des modes d'appropriation a pour objet de rendre la propriété publique responsable de ses profits et pertes.

Li Yining, Réflexions pour la réforme

[Grâce aux sociétés de capitaux, l'individu] s'affranchit lui-même de sa propriété tout en retenant le bénéfice. Jean Jaurès, De la propriété socialiste

L'enjeu est clair. Réformer les entreprises d'État impose-t-il de les privatiser? Si non, comment moderniser leur gestion tout en préservant les droits patrimoniaux de la nation chinoise sur leurs actifs? L'adoption de techniques de droit privé apporterait-elle les solutions et les garanties recherchées? Quelle place un droit « socialiste » peut-il octroyer à de telles techniques, à emprunter de surcroît le plus souvent, soit au droit chinois républicain, soit aux droits étrangers?

À l'énoncé de telles questions on comprendra que la réforme du système d'appropriation des moyens de production est au centre même de la réflexion actuelle sur la réforme du système économique chinois et de son secteur d'État. Dans ce débat, les intervenants les plus modernistes puisent leur inspiration dans les travaux d'économistes états-uniens tel Ronald Coase lauréat en 1991 du prix Nobel d'économie. Le moindre des paradoxes n'est

1 Thierry Pairault est Directeur de recherche au Centre National de la Recherche Scientifique ; il enseigne à l'École des Hautes Études en Sciences Sociales et à l'Institut National des Langues et Civilisations Orientales. 
pas qu'un chantre du libéralisme puisse influencer des économistes ayant tété le sein du marxisme, mais qu'une doctrine ultra-libérale partageât avec l'orthodoxie marxiste la même conviction que le droit de propriété est l'ultima ratio de l'économie politique. Postulat ambigu à telle enseigne que les Chinois ont cru devoir rappeler l'antériorité de la formulation de Marx sur celle de Coase $^{2}$ !

\section{La propriété d'État entre clarification et protection}

Dans la théorie classique pré-coasienne, qui était celle d'Arthur C. Pigou ${ }^{3}$, le processus de prise en compte de certains coûts s'opérait par le biais d'une intervention de la puissance publique. Or, pour des raisons historiquement différentes, les économistes libéraux et certains économistes chinois tombent aujourd'hui d'accord pour estimer que cette solution est peu ou insuffisamment efficace ; c'est pourquoi les premiers entendent lui substituer et les seconds veulent lui adjoindre un autre mécanisme.

La théorie défendue par Coase dans sa fameuse étude de $1960^{4}$ expose que le problème des coûts externes, ou externalités pour contrefaire un idiotisme d'économistes anglo-saxons, est fondamentalement un problème d'appropriation des coûts donc de définition des "droits de propriété " (property rights, i.e. droits patrimoniaux relatifs à la propriété). L'expression externalities s'est spécialisée pour désigner le plus souvent des coûts, bien qu'elle désigne originellement des bénéfices (external profit) et des coûts (external cost) apparaissant lors d'un échange mais conçus hors de l'échange lui-même :j'achète un champ ; la présence des abeilles de l'apiculteur voisin

2 Cf. Yan Qifa, « Chanquan wenti yanjiu zongshu », Zhongguo qiye guanli nianjian 1996, Pékin, 1996, p. 398-401.

3 Arthur Cecil Pigou (1877-1959) est un économiste britannique de l'école dite de Cambridge. Il a été la tête de file des économistes "classiques" entre les deux guerres mondiales. Son ouvrage le plus connu a pour titre The Economics of Welfare ; paru en 1920, il traite précisément du problème des externalités que j'évoque ci-après.

4 Ronald H. Coase, "The Problem of Social Cost », The Journal of Law and Economics, 1960 (3), p. 1-44. 


\section{Droit de propriété et réforme du secteur d'État}

qui aident à la pollinisation de mes plantations est un avantage qui n'aurait pas été pris en compte dans l'évaluation du prix du champ; de même la pollution de l'usine voisine qui nuit à mes plantations serait un coût ignoré ; ces situations résulteraient d'une défaillance fonctionnelle du marché (market failure) à appréhender tous les phénomènes. Ce raisonnement peut s'appliquer aux coûts gérés par l'État au bénéfice de la collectivité comme la lutte contre la pollution ou la protection sociale.

Faute, donc, aux agents économiques d'internaliser les externalités ${ }^{5}$, le marché faillirait partiellement à accomplir sa mission régulatrice. Notons en passant que la réforme de l'unité de travail en Chine, la fameuse danwei, accomplit aujourd'hui au nom du marché le miracle d'externaliser des coûts la protection sociale - qui avaient été internalisés depuis fort longtemps ! Pour en revenir à l'école coasienne, s'il existe un marché libre sur lequel puissent s'échanger les droits de propriété, si leur contenu est clairement déterminé et si leur application est rigoureusement respectée, on obtient alors la structure économique la plus efficace - quelle que soit par ailleurs la distribution des richesses entre les individus. La théorie des « droits de propriété », telle qu'elle a été développée par des économistes comme Armen Alchian ou Harold Demsetz, explique également les différences dans les modes d'organisation sur la seule base des stimulants individuels résultant des rapports de propriété. Les droits de propriété spécifient les relations économiques et sociales que doivent observer les individus entre eux dans leur usage de ressources rares. Une telle appréhension du rôle de la propriété a souvent conduit les auteurs à une apologie sans nuance de la petite entreprise.

Cette théorie, pour être vérifiée, suppose en effet que le propriétaire d'un bien puisse exercer un contrôle réel sur celui-ci ou, pour le moins, que le représentant mandaté par le propriétaire agisse uniquement pour le compte de ce dernier. Cette théorie ne reconnait donc pas - du moins dans sa version initiale - que le mode de fonctionnement des entreprises privées modernes puisse aujourd'hui reproduire celui des entreprises publiques car les deux modes d'appropriation - privé et public — introduisent une séparation entre la propriété effective et la direction effective de l'entreprise avec pour conséquence

5 «To internalize the externalities». 
des divergences entre les intérêts des dirigeants effectifs et ceux des propriétaires. Aussi, pour juger de la rentabilité ou de l'efficacité d'une entreprise, la question préalable à laquelle il convient de répondre n'est pas tant celle du mode d'appropriation de l'entreprise que celle du contrôle exercé sur les dirigeants et, plus largement, sur tout le personnel de l'entreprise. C'est le problème de l'insider control étudié notamment par Aoki Masahiko dont les travaux ont été traduits et publiés en Chine dès $1995^{6}$.

La réévaluation des politiques - prônée par Zhu Rongji et entérinée en mars 1998 par Li Peng dans son rapport à la IX ${ }^{\mathfrak{e}}$ Assemblée nationale populaire - donnait à cette approche une nouvelle actualité. Jiang Zemin, lors de la réunion du Xve Congrès du PCC en septembre 1997, avait revendiqué une «clarification des droits de propriété » (caiquan qingxi), en accordant la priorité à la transformation des entreprises d'État en sociétés de capitaux (gufenhua) ${ }^{7}$. Très rapidement ce mot d'ordre en faveur d'une actionnarisation à marche forcée a perdu de sa prééminence devant les dysfonctionnements qu'il générait en particulier dans les entreprises d'État les plus petites : accaparement de parts au profit d'une minorité, détournement d'actifs d'État, extorsion de l'épargne des employés... ${ }^{8}$ Plus grave encore, s'est développée une frénésie de ventes d'entreprises d'État ("mai qiye" $z h i$ feng), d'une ampleur telle que le Conseil des affaires de l'État a dû, pour la

6 Le livre dont il a dirigé la publication en collaboration avec Kim Hyung-Ki, Corporate Governance in Transitional Economics: Insider Control and the Role of Banks, World Bank, 1995, a été traduit et publié en Chine la même année sous le titre suivant: Zhuangui jingii zhong de gongsi zhili jiegou: neiburen kongzhi he yinhang de zuoyong, Beijing, Zhongguo jingji chubanshe,1995. Notons, inter alia, la traduction de l'ouvrage de Yoram Barzel (théoricien appartenant à une tendance "dure "), Economic Analysis of Property Rights, New York, Cambridge University Press, 1989, sous le titre suivant : Caichan de jingji fenxi, Shanghai, Shanghai renmin chubanshe/ Shanghai sanlian shudian, $1997,171 \mathrm{p}$.

7 Le discours de Jiang Zemin au Congrès peut être consulté sur le site Internet du Quotidien du peuple à l'adresse suivante : http://www.snweb.com/gb/ people_daily/1997/09/13/0913b.htm.

8 Cf. le rapport de Li Peng présenté lors de la réunion de la $\mathrm{IX}^{\mathrm{e}}$ Assemblée nationale populaire en mars 1998. 


\section{Droit de propriété et réforme du secteur d'État}

contenir, intervenir en juillet 1998. Un éditorial du Quotidien du Peuple du 20 novembre 1998 fustige certaines pratiques supposées réformatrices et explicite ainsi la politique gouvernementale en matière de réforme des entreprises d'État :

[...] Ces dernières années, de nombreuses et spécieuses « théories 》 de la réforme des entreprises sont en vogue. Parmi celles-ci on note que " pour lever les difficultés des entreprises d'État $y$-a-qu'à réformer le système de propriété ", « $y$-a-qu'à répartir entre chacun les actifs pour tout dynamiser ", « $y$-a-qu'à vendre les petites entreprises d'État pour les réformer $»$. Ces vues ne tiennent pas debout d'un point de vue théorique et ont montré leur inanité dans la pratique. Les origines des difficultés actuelles des entreprises d'État sont multiples [sur-investissement, interventionnisme, manque d'autonomie, manque de compétitivité, travailleurs surnuméraires, gestion incompétente] et aggravées par une sous-capitalisation et un surendettement - sans parler de l'influence de la crise financière internationale. Ces problèmes sont sans rapport aucun avec le système de propriété et ce n'est certainement pas la vente à des particuliers qui les résoudra [...] [Certains dirigeants locaux] croient dur comme fer que "l'actionnariat fait revivre ", que « la vente fait revivre ", ou encore considèrent que les petites entreprises d'État sont un « fardeau » et donc qu'il convient de s'en débarrasser par la vente. Le résultat est que non seulement les entreprises n'ont pas été revitalisées, mais encore que les travailleurs ont été mis à la rue; de fait on ne s'est pas débarrassé du « fardeau $»[. . .]^{9}$

Ce que recherchent les dirigeants chinois n'est pas tant la privatisation du secteur étatique que la dématérialisation des actifs d'État ; de possesseur de biens matériels tangibles, l'État deviendrait détenteur de droits sur ces biens ou sur leur contrepartie quand ils ont été vendus, échangés, fusionnés... Pour réussir cette opération il importe donc, d'une part, d'éviter les détournements d'actifs, et d'autre part, de constituer des équipes de dirigeants d'entreprise

9 On trouvera l'éditorial à l'adresse suivante : http://www.snweb.com/gb/ people_daily/1998/11/20/a1120001.htm ainsi qu'une traduction en français à l'adresse suivante : http://www.ifrance.com/gec/Notes/RMRB981120.htm. L'emploi de l'expression orale " $y$ - $a-q u$ 'à " est destiné à rendre moins la lettre que l'esprit du commentateur chinois. 


\section{Thierry Pairault}

compétents sur lesquels le gouvernement puisse exercer un contrôle effectif. Au regard de ces exigences, le réajustement des modes d'appropriation des moyens de production devient secondaire tant que la loi organise et garantit la protection des droits de l'État à la propriété des actifs qu'il détient aujourd'hui.

\section{Le droit de propriété et sa définition}

Le droit de propriété est défini par l'article 71 des Principes généraux $d u$ droit civil ${ }^{10}$ au chapitre premier du Titre $5:$ «Du droit de propriété sur les biens et des droits patrimoniaux en rapport avec le droit de propriété » (Caichan suoyouquan he yu caichan suoyouquan youguan de caichan quan). Traduire caichan quan par "droits patrimoniaux » est une approximation suggérée par l'expression chinoise. Le droit français subdivise les droits patrimoniaux en deux catégories, les droits sur les choses ou " droits réels » et les rapports juridiques entre deux sujets de droit ou « droits personnels », lesquels constituent les obligations au sens technique. Si les juristes chinois de l'époque républicaine semblent avoir hérité de cette vision, certains juristes chinois actuels, auraient tendance à opposer les caichan quan - qui ne seraient pas des droits réels (wu quan), puisque ignorés par les Principes généraux du droit civil - aux zhaiquan - qui ne se confondraient pas davantage avec les droits personnels car les Principes généraux du droit civil incluent, parmi les obligations, les hypothèques, gages, et autres antichrèses (art. 89) ${ }^{11}$. Cette prise de position pourrait témoigner d'une influence du droit allemand qui considère que les obligations recèlent un double rapport (personnel et réel) : c'est le débiteur qui doit au créancier mais c'est le patrimoine qui répond de la dette. C'est ce qui expliquerait que

10 Le texte de 1986, les commentaires de la Cour suprême et les textes d'application ont été réunis in Liang Shuwen et al. (éd.), Minfa tongze ji peitao guiding xin shi xin jie, Pékin, 1996, 1146 p.

11 Le deuxième chapitre du Titre 5 des Principes généraux du droit civil traite des " obligations". 
l'opposition chinoise entre caichan quan et zhaiquan ne recouvre pas la distinction classique entre « droits réels » et « droits personnels ».

\section{La vision actuelle des droits patrimoniaux et des obligations}

\begin{tabular}{|c|c|}
\hline $\begin{array}{l}\text { droits patrimoniaux } \\
\text { droit de propriété } \\
\text { droits patrimoniaux en rapport } \\
\text { avec le droit de propriété }\end{array}$ & $\begin{array}{l}\text { caichan quan } \\
\text { suoyouquan } \\
\text { yu caichan suoyouquan } \\
\text { youguan de caichan quan }\end{array}$ \\
\hline $\begin{array}{l}\text { obligations } \\
\text { obligations stricto sensu } \\
\text { sûretés }\end{array}$ & $\begin{array}{l}\text { zhai quan } \\
\quad \text { zhai quan } \\
\text { zhai de danbao }\end{array}$ \\
\hline
\end{tabular}

D'une manière plus générale, il existe un débat sur le sens exact de l'expression caichan quan souvent rendue par celle de chan quan. Selon certains auteurs, tenants d'un libéralisme pur et dur, elle traduirait l'expression anglo-saxonne "property rights " ${ }^{12}$, et d'énumérer toute une série de définitions - celle de Coase mais aussi celles d'Alchian, Berle, Demsetz et d'autres encore qu'ils opposent à celle de Marx. De fait les conceptions des premiers contredisent celle du dernier. Ceux-là fondent leur doctrine sur un dogme anti-étatique et une vision « contractualiste » des rapports humains telle qu'elle implique exclusivement une appropriation privée. Celle de Marx se fonde sur une théorie du développement des forces productives conduisant inéluctablement à une appropriation collective des moyens de production. Les relations patrimoniales sont, pour les uns, le fruit de rapports contractuels entre individus, pour l'autre, le produit de l'histoire. Ces conceptions s'excluant mutuellement de façon si catégorique, il est clair que la politique de réforme du secteur d'État en Chine doit s'interpréter prioritairement à la

12 Certains la définissent même par ces deux seuls mots éludant toute explication; cf. Wang Kaiguo (éd.), Zhongguo qiye chanquan jiaoyi shiwu wenda, Pékin, 1995 , p. 1. 
lumière du système marxiste - du moins tant que la Chine restera " populaire » - , toute complaisance à l'égard de la théorie des « property rights ${ }^{13}$ ne se justifiant que par la réflexion qu'elle nourrit et non par l'éventuelle formulation d'une quelconque politique de privatisation.

La traduction socio-économique pro-libérale écartée pour l'heure, restent nombre d'interprétations plus juridiques. Pour certains auteurs, l'expression chan quan désignerait lato sensu le droit de propriété et les obligations tandis qu'elle serait stricto sensu équivalente à zhai quan. Pour d'autres, sa signification pourrait varier selon l'agent économique auquel elle s'applique ; elle se rapporterait tantôt au droit de propriété, tantôt à un droit de détention accompagné d'un droit administration - cas des entreprises d'État ${ }^{14}$. Ces hésitations terminologiques ne sont pas étonnantes puisque les instances suprêmes elles-mêmes alimentent la confusion en identifiant souvent caichan quan et suoyouquan ${ }^{15}$. Mais, nous explique-t-on par ailleurs ${ }^{16}$, c'est ainsi que doit se comprendre une affirmation de Marx dans sa Contribution à la critique de l'économie politique. L'économiste Sun Yefang était certainement plus convaincant quand il suggérait, en 1956, que les traductions successives de l'allemand au russe puis au chinois pourraient être à l'origine de ces ambiguités terminologiques ${ }^{17}$.

13 Les partisans de cette théorie sont dits appartenir à l'École des « property rights 》 (chanquan xuepai).

14 Wang Kaiguo, op. cit., p. 3-5.

15 Ajoutons que caichan quan est souvent équivalent à zichan quan. De surcroît, pour les uns caichan quan et caichan suoyouquan sont synonymes tandis que pour les autres - tenants des thèses " coasiennes "- ces deux expressions doivent respectivement être la traduction de « property rights » et de " ownership right ".

16 Cf. inter alia Zhang Zhiguang et alii, « Guanyu faren caichan quan de jige wenti », Dongbei shida xuebao, 1995, n 5 , p. 31-32.

17 Sun Yefang, "Lun zuowei zhengzhi jingjixue duixiang de shengchan guanxi » (Des rapports de production comme objet de l'économie politique), repris dans Sun Yefang xuanji, Taiyuan, 1984, p. 395-396. 


\section{Droit de propriété et réforme du secteur d'État}

\section{Les quatre attributs du droit de propriété}

Quoi qu'il en soit, le droit de propriété se définit pour le propriétaire d'une chose comme le droit à jouir de quatre prérogatives (quanneng), dont trois constituent les attributs classiques de la propriété au sens du droit français mais dont la quatrième peut ouvrir la porte à de subtiles interprétations. Les trois attributs classiques sont ceux que résume la litanie usus, fructus, abusus - soit en chinois shiyong, shouyi, chufen - avec une nuance toutefois puisque la conception française du droit de jouir de sa chose ne se confond pas entièrement avec la chinoise. Pour la française, la jouissance pour un propriétaire consiste dans le droit de faire (ou de ne pas faire) fructifier sa chose, d'en percevoir (ou de n'en pas percevoir) et d'en consommer (ou de n'en pas consommer) les fruits. Pour la chinoise, ce droit se comprend davantage comme le droit d'en tirer profit ; cette interprétation est renforcée par l'emploi de l'expression zixi pour désigner les fruits. Le caractère $z i$ signifie " engendrer » (d'où " multiplier ») et le caractère $x i$, "intérêt », " profit », mais aussi « avoir des enfants » ${ }^{18}$. Cette conception n'encourage pas l'éventuel usufruitier d'un bien " à en jouir en bon père de famille " ${ }^{19}$; le zixi chinois fait irrévocablement penser à l'intérêt qu'Aristote condamne et baptise $\tau^{\prime} x o \zeta$ ce qui en grec signifie « rejeton $»{ }^{20}$.

18 Le sens étymologique serait « respirer ».

19 Même si, par ailleurs, le « jouir en bon père de famille " du Code civil français (art. 601) implique "avoir des enfants ", mais là n'est pas ce qu'entendait le législateur français qui s'inquiétait de la prudence dans la gestion du patrimoine.

20 La dimension morale que l'appellation chinoise ajoute est parfaitement illustrée par une coquille du Zhonghua faxue da cidian : minfaxue juan (Grand dictionnaire chinois de science juridique : droit civil) qui, sous la férule du grand juriste Tong Ruo, écrit (p. 893) niexi en lieu et place de zixi, le caractère nie signifiant « mauvais »! 


\section{Le droit civil républicain et les droits réels}

droits réels (wu quan)

droit réel plénier (wanquan wu quan) $\Rightarrow$ droit de propriété (suoyouquan)

droits réels précaires (dingxian wu quan)

$\Rightarrow$ droits réels usufructuaires (yongyi wu quan)

$\Rightarrow$ droits réels accessoires (sûretés) (danbao wu quan)

quasi-droit réel (leisi wu quan) $\Rightarrow$ possession (zhanyou)

La distinction classique entre le droit sur sa chose ( $z i$ wu quan), et le droit sur la chose d'autrui (ta wu quan), recouvre de fait celle entre « droit plénier » et « droits précaires $»$.

Le quatrième attribut du droit de propriété - le premier énoncé par l'article 71 des Principes généraux du droit civil — se lit zhanyou et peut se traduire, en reprenant une terminologie française, par « possession » ou par « détention ». En droit français, la possession est un état de fait ; le possesseur d'un bien peut, par coïncidence, en être le propriétaire, mais il peut tout aussi bien n'en être que le maître. En revanche, la détention à titre précaire est un rapport juridique ; le détenteur précaire a la maitrise effective d'un bien en vertu d'un titre qui identifie le propriétaire et reconnaît le droit de ce dernier sur le bien.

Le droit chinois républicain, encore qu'il distinguât ces deux notions et opposât la possession (zhanyou), à la détention (chiyou ou suochi), instaura la première en catégorie juridique formelle accessoire aux droits réels ${ }^{21}$. Ce faisant le droit républicain s'inspirait du droit japonais qui avait institué un droit à une forme de protection possessoire (sen-ȳuken, mot-à-mot « droit

21 Le Code civil taiwanais, reprenant celui de l'époque républicaine, a hérité de cette distinction. Shi Shangkuan dit qu'il ne faut pas confondre zhanyou et chiyou qui traduisent les expressions latines de possessio et detentio; il ajoute que la première est une possession de droit (falü shang zhanyou), en latin possessio civilis, tandis que la seconde est une possession de fait (shishi shang zhanyou), en latin possessio naturalis; cf. Shi Shangkuan, Wuquan fa lun, Taipei, 1957, p. $476-477$. 


\section{Droit de propriété et réforme du secteur d'État}

de possession »), qui se lit en chinois zhanyou quan, droit inscrit parmi les droits réels à côté du droit de propriété, shoyüken. Le droit républicain s'en démarquait néanmoins ; il se rapprochait davantage des positions doctrinales $\mathrm{du}$ droit allemand. En effet, le droit français considère que la possession est constituée de deux éléments. L'un matériel, le corps de la possession ou corpus (traduit en chinois par l'expression tisu) se manifeste par l'exercice d'un pouvoir effectif sur la chose possédée (duiyu wu you shishi shang guanling zhi li zhe, wei zhanyou ren dit l'article 940 du Code civil républicain). L'autre psychologique, l'esprit de possession ou animus (traduit en chinois par l'expression xinsu), témoigne d'une prétention au titre de propriétaire. Le droit allemand ne retient que l'élément matériel et peut en conséquence octroyer le bénéfice de la possession aux détenteurs à titre précaire (qui donc ne revendiquent pas la propriété de la chose possédée). Les juristes républicains pensaient trouver ainsi une voie intermédiaire entre le droit allemand et le droit japonais qui, tout en témoignant que la possession est un état de fait, assiérait au mieux le droit du possesseur ${ }^{22}$.

\section{L'introduction de l'attribut zhanyou}

La question qui se pose donc est de comprendre pourquoi les juristes chinois de Chine populaire éprouvèrent après 1949 la nécessité d'intégrer zhanyou comme attribut du droit de propriété. Si ce ne fut par interprétation dogmatique étroite pour rejeter toute réflexion émanant de prédécesseurs républicains ou japonais - , les motifs idéologiques n'en furent pas absents. L'influence des codes civils adoptés par la République de Russie ${ }^{23}$ en 1922 puis en 1964 est claire à la lecture des articles 58 et 92 . Cette adoption à

22 Une excellente présentation du problème apparaît chez Shi Shangkuan, op. cit., p. 67-78, et p. 476-489. Ajoutons que la pratique de nantissements entraînant une dépossession du débiteur - gage (cas des biens mobiliers) zhi quan, et antichrèse (cas des biens immobiliers) dian quan - rend plus que nécessaire de fixer les règles applicables au transfert de la possession.

23 J'entends la République socialiste fédérative soviétique de Russie et non l'URSS. 


\section{Thierry Pairault}

l'aveugle est indirectement avouée par Wang Liming dans son ouvrage publié en 1992 où il consacre un chapitre à étudier le sens de zhanyou et de ses relations avec le droit de propriété ${ }^{24}$ : «Bien que les droits [français et allemand] ne distinguent pas la possession (zhanyou), comme un attribut du droit de propriété, tous (sic) les auteurs s'accordent pour le considérer comme tel ». Et Wang Liming d'en référer à l'autorité du célèbre juriste émigré à Taiwan Shi Shangkuan, avant de continuer ainsi : "Les codes civils de l'URSS et des pays de l'Europe de l'Est proclament tous que le droit de possession (zhanyou quan), est un attribut du droit de propriété. " ${ }^{25}$ Comme justification, c'est un peu mince ${ }^{26}$; essayons d'assembler les éléments épars donnés en réflexion.

Commençons par une explication intéressante que je me contenterai de mentionner puisqu'elle omet de prendre en compte les origines historiques du concept, en particulier l'influence soviétique qui attribue la nécessité d'inclure zhanyou comme attribut du droit de propriété, au seul fait que le droit civil chinois «populaire » ne retient pas la notion de droits réels. En conséquence, les juristes auraient été contraints de lui substituer un système palliatif supposant cette inclusion en imposant, en 1992, l'explicitation qu'offrent les quatorze critères d'autonomie des entreprises d'État ${ }^{27}$. Sans rejeter a priori cette justification, je noterai que si elle est avérée pour la Chine, elle l'est également pour l'URSS ; la question reste donc pendante.

Les rapports de production - c'est-à-dire les rapports qu'établissent les hommes entre eux au cours du processus de production - se modifient au fur et à mesure que se développent les forces productives matérielles, nous apprend la vulgate marxiste. En d'autres termes, la nature des rapports de

24 Wang Liming, Gaige kaifang zhong de minfa yinan wenti, Changchun, 1992, p. 122-131.

25 Ibid., p. 126-127.

26 D'autres auteurs ne se donnent même pas cette peine comme ceux ayant rédigé le manuel de droit civil de l'Université de Pékin en 1982 : Beijing daxue shiyong jiaocai : minfa jiaocheng [Matériaux pédagogiques expérimentaux : manuel de droit civil], Pékin, Beijing daxue chubanshe, 1982, p. 131 sq.

27 Wang Xiaolong et al., « Guoyou qiye chanquan yu xiandai qiye zhidu », Xiandai qiye baodao, $\mathrm{n}^{\circ} 167,1997$ (5), p. 58-60. 


\section{Droit de propriété et réforme du secteur d'État}

production résulte de l'état et du progrès des forces productives elles-mêmes. Les rapports patrimoniaux ou rapports de propriété (chanquan guanxi), sont quant à eux, l'expression juridique - abstraite - de ces rapports de production (shengchan guanxi) ; leurs formes évoluent en suivant les transformations des forces productives et des rapports de production correspondants. C'est ainsi que se comprend ce passage de la préface de Marx à sa Contribution à la critique de l'économie politique que citent systématiquement les commentateurs chinois et dont je donne une des traductions françaises :

À un certain stade de leur développement, les forces productives matérielles de la société entrent en contradiction avec les rapports de production, ou, ce qui n'en est que l'expression juridique, avec les rapports de propriété au sein desquels elles s'étaient mues jusqu'alors ${ }^{28}$.

Parce que les pays socialistes entendent ${ }^{29}$ résoudre cette contradiction, leur système de propriété se fonde sur l'appropriation publique des moyens de production - les principales formes de propriété étant la propriété étatique et la propriété collective, il y a socialisation des moyens de production et non nationalisation ${ }^{30}$, ni non plus étatisation. Par suite, le concept de droit de propriété inscrit dans les codes civils de ces pays ne pourra qu'être assez différent d'un concept du droit de propriété des autres pays fondé sur une appropriation privée. L'erreur ici serait donc de confondre la forme de l'appropriation (zhanyou xingshi ou suoyouzhi xingshi), avec l'appropriation effective (zhanyou) des moyens de production, comme l'aurait en partie fait, nous dit-on ${ }^{31}$, le grand juriste soviétique A.V. Venediktov, auteur en 1948

28 Karl Marx, Contribution à la critique de l'économie politique, Paris, Éditions sociales, 1977 , p. 3.

29 Sans doute l'emploi d'un des temps du passé conviendrait mieux pour évoquer certains de ces pays.

30 La socialisation n'exclut pas la nationalisation quand la première aboutit à ramener dans le giron national un bien qui était aux mains de détenteurs étrangers.

31 C'est ce qu'affirme Wang Liming, « Guojia suoyouquan de falü tezheng yanjiu », Falï kexue, 1990, n 6, p. 31-32. 


\section{Thierry Pairault}

d'un ouvrage remarqué sur la propriété étatique socialiste ${ }^{32}$. La propriété étatique ou, plus exactement, du peuple entier (quanmin), sera donc l'expression juridique de l'appartenance à la société entière des moyens de production mais non celle de leur gestion effective.

\section{Appropriation et administration}

Cette remarque conduit directement au problème de l'organisation de la société socialiste et à celui de la séparation - acceptée - dans le secteur étatique entre, d'une part, l'affirmation par l'État de son droit à la propriété des moyens de production et, d'autre part, leur administration effective par des unités de production. En 1961, l'économiste Sun Yefang écrivait dans un rapport intitulé « Le problème du système de gestion au sein de l'économie relevant de la propriété du peuple entier » :

Sous le régime de la propriété du peuple entier, les droits d'appropriation (zhanyou), d'usage et de disposition constituent un ensemble; le droit de propriété en constitue un autre. Les organismes d'État ont le droit de s'approprier, d'user et de disposer des actifs d'État qui leur ont été alloués à la condition de se conformer aux prescriptions qui sont les leurs. En revanche le propriétaire de ces actifs est l'État ${ }^{33}$.

Sun Yefang, reprenant Marx, oppose donc le droit de propriété stricto sensu (c'est-à-dire à qui, appartient tel bien, par opposition au droit de propriété lato sensu qui s'identifie à sa définition en droit civil ${ }^{34}$ ) à l'ensemble de trois autres droits (droits d'appropriation, d'usage et de disposition (zhanyou, shiyong he zhipei quan ${ }^{35}$ ) que Sun Yefang nomme « droit d'administration »

32 Je fais référence aux thèses de Venediktov, doyen de la faculté de droit de l'Université de Léningrad, telles que les révèlent les exégèses chinoises qui, ici du moins, importent plus que le texte original. Le titre de son ouvrage se lit Gosudarstvennaja sotsialistitcheskaja sobstvennost.

33 Sun Yefang xuanji, op. cit., Taiyuan, 1984, p. 241

34 Cf. Zhang Zhiguang, op. cit., p. 31 sq.

35 Ceci est la formulation " classique " par laquelle les Chinois traduisent le texte de Marx. Aujourd'hui, l'expression zhipei est le plus souvent rendue par chufen. 


\section{Droit de propriété et réforme du secteur d'État}

(jingying quan), suivant en cela les théoriciens soviétiques. Notons dans l'énoncé l'absence du droit aux fruits dont on nous dit que, pour Marx, il se déduit du droit d'usage - à rebours du droit français pour le lequel le jus utendi se déduit du jus fruendi ${ }^{36}$.

L'introduction de zhanyou comme attribut du droit de propriété lato sensu et son inclusion dans un droit d'administration se justifiait donc pour des raisons d'orthodoxie doctrinale, mais aussi pour des raisons d'organisation sociale. Aujourd'hui, avec le retour à une forme d'économie de marché et avec l'introduction de nouvelles formes d'entreprise, le risque n'est plus seulement que l'État-propriétaire perde en partie son contrôle sur la gestion de ses actifs mais qu'il soit bel et bien dépouillé de ses actifs et ce d'autant plus facilement que ceux-ci sont de plus en plus souvent dématérialisés. Par suite, zhanyou va servir à défendre les droits des parties intéressées, l'État propriétaire d'actifs et l'entreprise détentrice d'actifs d'État. Remarquons immédiatement que le système duel «droit de propriété/droit d'administration » a été conçu pour s'appliquer au secteur d'État, à la propriété d'État et aux entreprises d'État, excluant a priori les autres secteurs, les autres formes de propriété (propriété collective, propriété du citoyen ${ }^{37}$, propriété des groupes sociaux) et les autres types d'entreprise. Or, la constitution de sociétés de capitaux hors du seul secteur d'État tend à reproduire la séparation entre propriétaires et administrateurs dans les autres secteurs, à l'instar des pays capitalistes. Aussi, l'élaboration d'un droit d'administration spécifique aux entreprises d'État ne devrait plus constituer à terme qu'une péripétie juridique dès lors que la dématérialisation des actifs d'État se généralisera.

Zhanyou signale une détention précaire et, partant, protège les droits de l'État sur ses propres actifs. Contrairement au droit républicain, qui reconnaît l'acquisition de la propriété par voie d'usucapion ou «prescription acquisitive ${ }^{38}$ (qude shixiao), le droit chinois actuel ne l'autorise pas et ce

36 « La propriété est le droit de jouir et de disposer des choses de la manière la plus absolue, pourvu qu'on n'en fasse pas un usage prohibé par les lois ou les règlements " dit l'art. 544 du Code civil français.

37 Propriété du citoyen - donc chinois - et non propriété privée, cf. infra.

38 Usucapion, littéralement " prendre (capere) l'usage (usus) », est le terme exact 


\section{Thierry Pairault}

d'autant moins que l'histoire lui apprend que les instances judiciaires suprêmes - en l'espèce le Yuan judiciaire (Sifa yuan) - ont reconnu en 1938 , en 1941 puis en 1945 , aux personnes physiques comme aux personnes morales le droit d'acquérir la propriété de biens publics par le biais d'une simple possession ${ }^{39}$. Cette protection de la propriété d'État est renforcée par l'article 73 des Principes généraux du droit civil qui, à défaut de donner une définition de cette propriété, constate son caractère sacré, d'où l'interdiction d'y porter atteinte : " guojia caichan shensheng bu ke qinfan ». Cette défense vigilante de la propriété d'État est aussi assurée par l'article 79 des Principes généraux du droit civil qui se refuse à considérer que des biens mobiliers puissent n'appartenir à personne (res nullius). Au pire sontils « sans maître " (res derelictea) ${ }^{40}$; s'il est impossible d'établir l'identité du propriétaire, ces biens reviennent à l'État sans bénéfice pour leur inventeur ou occupant. L'occupation ne pouvant jouer en matière mobilière, elle ne s'appliquera a fortiori pas davantage en matière immobilière; les biens immobiliers appartiennent de plano à l'État aux termes de l'article 9 de la Constitution chinoise. Il est clair que la définition du droit de propriété est telle qu'elle permet de protéger l'État contre toute atteinte, tout trouble à sa jouissance, sans qu'il n'ait jamais à démontrer le bien fondé de ses prétentions.

Zhanyou manifesterait aussi une forme de protection possessoire afin que les entreprises d'État puissent défendre leur droit à détenir des actifs d'État, partant, défendre les droits de l'État-propriétaire sur ces actifs. Toutefois, les articles 82 (droit d'administration) et 117 (responsabilité de celui qui porte atteinte au patrimoine d'autrui) des Principes généraux $d u$ droit civil n'établissent pas explicitement le droit pour les entreprises d'État à intenter des actions destinées à remédier à un trouble possessoire ; aussi certains auteurs réclament-ils l'instauration d'un droit, pour les entreprises

même si les deux expressions sont depuis longtemps devenues synonymes. Pour une présentation de la prescription acquisitive en droit républicain, cf. Shi Shangkuan, op. cit., p. 63 sq.

39 Li Xiangru (éd.), Taiwan wuquan fa, Pékin, 1993, p. 29-30.

40 Notons l'ambiguité de l'expression chinoise wuzhu, qui peut tout aussi bien signifier « sans propriétaire » que « sans maître ». 


\section{Droit de propriété et réforme du secteur d'État}

d'État, à exercer des actions possessoires (zhanyou zhi su) à côté du droit, pour l'État, à exercer une action pétitoire (suoyou zhi su) ${ }^{41}$.

\section{Propriété étatique, propriété non étatique, propriété privée}

Face à cette propriété d'État surprotégée, la propriété privée fait pâle figure. Il faut d'abord noter que l'expression même de propriété privée (siyou) est bannie de la littérature juridique au profit de formules diverses qui ne sont pas que de simples euphémismes. Ainsi à la propriété publique (gongyou), oppose-t-on la propriété non publique (fei gongyou), concept plus large que celui de propriété privée car il juxtapose toutes formes d'appropriation qui ne sont ni étatiques ni collectives. De même, en concurrence avec les entreprises d'État (guoyou qiye, mot à mot «appartenant à l'État »), prospèrent nombre d'entreprises dont certaines sont des entreprises gérées privativement (siying qiye) et non des « entreprises privées», distinction qui n'est pas non plus sans signification. À Taiwan, où l'on n'a pas échappé à la mode des privatisations, on distingue très clairement la propriété effective des entreprises (gongyou qiye/minjian qiye, entreprise publique/entreprise privée) de leur mode de gestion (gongying qiye/minying qiye, entreprise gérée publiquement/privativement) et, très logiquement, il $\mathrm{y}$ existe des entreprises publiques dont la gestion est confiée à des capitaux privés (gongyou minying qiye).

La seule appropriation privée qui soit nommément garantie par la Constitution chinoise, dans son article 13, et par les Principes généraux $d u$ droit civil, dans son article 75 , est la propriété individuelle du citoyen (gongmin geren caichan suoyouquan), qui est restreinte à l'appropriation privative par les seuls détenteurs de la nationalité chinoise des revenus de leur travail et de leur épargne ainsi que des biens - principalement de consommation - qu'ils peuvent acquérir. Quelles sont les conséquences d'une telle définition?

41 Cf. Wang Liming, " Suoyouquan minfa baohu de ruogan wenti », Falü xuexi yu yanjiu, 1990, n $^{\circ} 4$, p. 57-62. 


\section{Thierry Pairault}

L'article 33 de la Constitution chinoise déclare que toute personne ayant la nationalité chinoise est un citoyen chinois (gongmin). Et certains commentateurs chinois de constater que les Principes généraux du droit civil opèrent une confusion entre « citoyen » et « personne physique » (ziran ren), excluant par suite les étrangers du cadre légal - i.e. tel qu'il est défini par les Principes généraux du droit civil - et donc, a priori, du droit à la propriété. Le problème est très logiquement le même pour les entreprises à capitaux étrangers ; celles-ci ont effectivement un statut légal, lequel ne leur donne toutefois pas le droit d'accéder à la propriété puisque les formes d'appropriation que ces entreprises supposent ne sont pas formellement inscrites dans le Titre 5 des Principes généraux du droit civil. La conséquence pratique est que ni le citoyen chinois ordinaire, ni une entreprise chinoise ordinaire (i.e. non spécifiquement habilitée) n'ont le droit de signer un contrat avec un investisseur étranger ${ }^{42}$.

Régimes juridiques et modes d'appropriation

ENTREPRISES RELEVANT DU DROIT COMMUN ANTÉRIEUR

1 Entreprises industrielles sous le régime de la propriété du peuple entier (loi de 1988)

2 Entreprises collectives de bourgs et de villages (loi de 1991)

3 Entreprises collectives rurales (loi de 1990)

4 Entreprises « privées » :

- Entreprises individuelles (Principes généraux du droit civil de 1986)

- Entreprises coopératives (loi de 1997)

42 Cf. l'interview du professeur Zhang Gengming dans Yuedan faxue (=The Taiwan Law Review), n²9, 1997, p. 80-84 
ENTREPRISES RELEVANT DE LA LOI SUR LES SOCIÉTÉS DE CAPITAUX (1993)

1 Sociétés de capitaux (droit commun)

- Sociétés à responsabilité limitée

- Sociétés anonymes par actions

2 Société à capital entièrement étatique (Titre 2, chapitre 3 de la Loi sur les sociétés)

3 Société à la constitution de laquelle participent des investisseurs étrangers (loi de 1995)

4 Entreprises unipersonnelles (loi d'août 1999)

ENTREPRISES À CAPITAUX ÉTRANGERS

1 Entreprises à capitaux entièrement étrangers (loi de 1986)

2 Entreprises à capitaux mixtes (loi de 1979)

3 Entreprises en coopération (loi de 1988)

Cette propriété privée est de surcroît bridée dans l'objet même auquel elle s'applique. Outre les revenus individuels et les biens de consommation, seuls « les biens de production nécessaires aux foyers paysans et aux travailleurs individuels pour entreprendre des activités à titre individuel » peuvent être appropriés à titre privatif ${ }^{43}$. C'est pourquoi Jin Shuping ${ }^{44}$, le président de la Fédération panchinoise de l'industrie et du commerce, qui représente le secteur privé, avait déjà entrepris, à l'occasion de la réunion de l'Assemblée nationale populaire en mars 1998, une campagne en faveur d'un amendement de la Constitution chinoise afin que soit reconnue et protégée la propriété privée et clarifié le statut légal des entreprises privées ${ }^{45}$. Cette clarification

43 Liang Shuwen, op. cit., p. 486-487.

44 Jin Shuping, âgé de plus de 80 ans, est un «capitaliste " shanghaien prérévolutionnaire ; il a participé au Forum économique mondial qui s'est tenu à Davos en février 1998.

45 " Private property ownership changes sought » et « Private business lobbies for same status and legal protection as state sector ", South China Morning Post (Internet Edition), 4 et 9 mars 1998. 


\section{Thierry Pairault}

paraît d'autant plus indispensable que, pour survivre et bénéficier des mêmes avantages que les entreprises d'État, nombre d'entreprises privées portent un « chapeau rouge » (hong maozi), c'est-à-dire ont été déclarées comme étant des entreprises d'État ; la simple rectification des appellations pourrait ne pas être suffisante car nombre de ces entreprises privées ont su profiter de leur chapeau pour «blanchir» en quantité les actifs d'État des danwei dont elles ont été proclamées parentes...

La révision constitutionnelle adoptée en mars 1999 ne tire pas son intérêt du seul amendement - largement commenté — de l'article 11 mais aussi de la réécriture de l'article 6 . Celui-ci, dans sa version initiale, stipulait que la base du système économique socialiste était l'appropriation publique des moyens de production. Dans sa version amendée, il est dorénavant précisé que cette appropriation publique est l'élément principal, d'autres modes d'appropriation pouvant toutefois jouer aussi un rôle concurrent au « stade initial du socialisme » - celui que l'on reconnaît désormais être celui de la Chine. Sans évoquer la propriété privée des moyens de production, cet amendement en suggère la légalité mais en limite la légitimité à ce fameux stade initial dont l'achèvement doit provoquer conséquemment celui de cette forme privative d'appropriation. Le nouvel article 11 signale cette promotion des activités relevant de la propriété non étatique — du grade de complément à celui d'élément constitutif —-mais se garde d'évoquer toute propriété privée, et ne fait état que «d'activités économiques individuelles, d'activités économiques menées à titre privé et autres activités entreprises hors du cadre de la propriété étatique ».

\section{Secteur d'État et privatisation}

Il est devenu un lieu commun de dire que le secteur d'État en Chine a perdu de son importance. Cette évolution s'est toutefois rarement accompagnée d'une privatisation des entreprises d'État à l'instar des politiques menées par divers gouvernements conservateurs européens dans les années 1970 et 1980, puis par les libéraux russes dans les années 1990. On assiste plutôt à une privatisation mesurée de l'économie puisque le nombre et la production des entreprises d'État n'ont cessé de croître ces dix dernières années : pour 


\section{Droit de propriété et réforme du secteur d'État}

les seules entreprises industrielles, le nombre d'entreprises d'État a cru d'un quart tandis que leur valeur ajoutée a plus que quadruplé.

Ce qui justifierait la privatisation des entreprises d'État serait leur relative inefficacité. Si la grande majorité des auteurs - chinois ou non s'accordent pour les juger peu rentables, rares sont ceux qui s'appuient sur des études réellement documentées. En règle générale, on nous expose que le rapport entre le capital investi et la production des entreprises d'État est moins favorable que celui qu'on établirait pour les entreprises collectives, les entreprises privées ou autres, oubliant qu'un tel indicateur supposé financier est essentiellement un indicateur technique révélant l'intensité capitalistique rendue nécessaire par l'activité même de telle ou telle catégorie d'entreprise. Des industries de main-d'œuvre (donc le plus souvent des entreprises collectives ou privées) auront une intensité capitalistique moindre que des entreprises (le plus souvent d'État) de plus grande taille, faisant appel à des techniques plus modernes et œuvrant dans l'industrie lourde ; par voie de conséquence, les premières paraîtront a priori toujours plus rentables que les secondes.

Souvent aussi, la démonstration consiste à rapporter l'investissement global (c'est-à-dire l'accroissement du capital et non plus son montant global) à l'accroissement de leur production (et non plus le montant de celle-ci). Le recours à un tel indicateur (nommé « coefficient de capital ») est tout autant une pétition de principe. Les entreprises de bourgs et de villages, dont il est de bon ton de proclamer l'efficacité, sont des entreprises de main-d'œuvre, donc globalement peu capitalistiques; elles auront obligatoirement un coefficient de capital plus favorable (faible) que les entreprises d'État, globalement plus capitalistiques; et celles-là paraîtront plus « rentables » que celles-ci ! Poursuivons l'analyse et opposons deux régions chinoises hypothétiques, dont l'une rurale développe des petites entreprises de maind'œuvre, et l'autre, plus urbaine, modernise ses entreprises d'État. Le modèle de croissance de Domar et Harrod nous apprend que, ceteris paribus, la région rurale aura un taux de croissance obligatoirement supérieur !

Parmi les arguments pour le moins contestables qui peuvent être avancés, est celui qui consiste à tirer des conclusions d'un indicateur rapportant la valeur ajoutée créée par chaque yuan dépensé en salaires, cotisations sociales et autres avantages sociaux. Un tel calcul montrera très normalement que les 


\section{Thierry Pairault}

petites entreprises privées sont plus rentables que les entreprises d'État puisque celles-là ont une structure de l'emploi récente (jeunes travailleurs non mariés...), versent des salaires peu élevés, ont de faibles charges sociales (absence de services sociaux, non-respect de leurs obligations...).

Le caractère global - et ce n'est pas là le moindre de leurs défauts des indicateurs généralement utilisés interdit toute analyse un peu fine, nuancée, que commande impérieusement toute évaluation objective. Il est clair que cette appréciation négative de la rentabilité des entreprises d'État chinoises est d'abord un jugement politique émis par des acteurs souvent non chinois puisque aussi bien la privatisation des entreprises d'État chinoises n'est pas une simple question de politique intérieure. Dans les pays en voie de développement, la privatisation des entreprises d'État signifie le plus souvent une dénationalisation, c'est-à-dire un transfert du contrôle de ces entreprises à des investisseurs étrangers. Là, comme en Chine, où l'appropriation par l'État a originellement été vécue comme une proclamation de sa propre existence, toute privatisation ne peut aujourd'hui apparaitre que comme une retraite sous la pression d'instances internationales. Plus une nation dépend de l'investissement étranger pour se moderniser (est-il nécessaire de rappeler son rôle en Chine ?) plus la privatisation de ses entreprises d'État risque d'être vécue comme une perte de souveraineté. Plus une nation dépend des prêts du Fonds monétaire international ou de la Banque mondiale (la Chine est l'emprunteur le plus important de cette dernière) ${ }^{46}$, plus elle sera sensible aux exigences de privatisations imposées par ces

46 Le montant cumulé de 1994 à 1999 des emprunts chinois s'élève à 16,6 milliards de dollars dont 2,1 au titre de la seule année $1999 ; 1,6$ milliards supplémentaires ont été budgétisés pour 2000. Sur les 16,6 milliards déjà octroyés, 12,6 milliards ont été effectivement employés et la Chine a déjà remboursé 2,3 milliards. Fin 1999 , le solde à honorer est donc de 10,3 milliards de dollars (ou encore 12,4 milliards si l'on inclut la dotation prévue pour 2000), soit un montant d'emprunt voisin du crédit ( 13 milliards de dollars) que virtuellement la Banque mondiale pourrait, selon ses propres calculs, concéder à la Chine (cf. les propos de JeanMichel Sévérino, Vice-président de la Banque mondiale en charge de la zone Asie orientale et Pacifique, rapportés par le South China Morning Post (Internet Edition), 25 septembre 1999). 


\section{Droit de propriété et réforme du secteur d'État}

institutions ${ }^{47}$. L'inefficacité souvent avérée de nombreuses petites entreprises d'État chinoises devint la raison d'envisager leur privatisation à toutes grandes ou petites : yu jia zhi zui, he huan wu ci, dicton dont l'équivalent serait « qui veut tuer son chien, l'accuse de la rage ». D'où la tendance à proclamer que les entreprises d'État ne faisaient que peu ou pas de profits.

\section{Privatisation et " société-sation"}

Malgré leur faible rentabilité, de nombreuses entreprises d'État, globalement, ne méritent donc pas les opprobres dont on les accablent, sauf à croire que le jeu soit de s'incliner devant les diktats du «consensus de Washington » 48 . Cette prudence permet au gouvernement chinois de recevoir l'aide du FMI

47 On trouvera des références à cette situation dans Paul Starr, «The meaning of privatization ", Yale Law and Policy Review, 1988 (6), p. 6-41.

48 Inspirée du discours de Joseph Stiglitz à Helsinki le 7 janvier 1998, l'expression « Washington consensus » désigne le credo ultra libéral que Washington, le FMI et la Banque mondiale, imposent aux pays en voie de développement de respecter pour bénéficier de leur soutien. Cet ancien conseiller de Bill Clinton, devenu vice-président et économiste en chef de la Banque mondiale, dans un moment d'humilité et d'une rare lucidité, faisait là une véritable autocritique, dénonçait un certain nombre de dogmes à la mode, constatait que l'économie de marché n'était pas la panacée, observait que les bénéfices des privatisations avaient été très surestimés, admettait que les dégraissages systématiques étaient nocifs, et proclamait que la réforme du système de propriété était secondaire. La faction la plus conservatrice accuse, quant à elle, les tenants du « consensus de Washington » d'être responsables du fiasco russe qui, dit-elle, aurait pu être évité si on n'avait pas tenté d'accompagner le « choc thérapeutique » (i.e. l'introduction forcée du mécanisme du marché) pour le rendre moins blessant ! Ce discours est disponible à l'adresse Internet suivante : http:/www.global policy.org/socecon/bwi-wto/ stig.htm. Il est très significatif que, de l'aveu même de la Banque mondiale, tout le discours pro-privatisation des entreprises d'État ne repose, faute de données, sur aucune analyse statistique sérieuse des conséquences d'une telle privatisation. Les statistiques portant sur quatre-vingt huit pays que vient de rassembler la Banque mondiale afin de constituer une nouvelle banque de données sur les 


\section{Thierry Pairault}

et de la Banque mondiale ainsi que les encouragements de Washington; dans le même temps il peut procéder au renforcement (à la " recomposition ») du secteur d'État en élaguant les entreprises les plus faibles, en dégraissant les effectifs et en changeant le régime juridique - la « société-sation » (gongsihua) - garantir à terme aux entreprises d'État une rentabilité et une compétitivité accrues. La réforme du secteur d'État en tant que tel - i.e. sa privatisation stricto sensu et non plus sa seule recomposition - apparaît d'autant moins impérieuse que la santé des entreprises à capitaux étrangers, fers de lance de la stratégie chinoise de développement, est souvent préoccupante.

Parce que les autorités chinoises adoptent des techniques de droit privé pour assurer une meilleure gestion des actifs d'État, nombreux sont ceux qui identifient la métamorphose des entreprises d'État en sociétés de capitaux (gongsihua), à leur privatisation (siyouhua) ; c'est le cas par exemple de Tam On Kit qui écrit dans un ouvrage paru en 1998 :

Bien qu'aujourd'hui l'agenda des autorités chinoises ne prévoit pas encore un plan de privatisation à grande échelle (en dépit des décisions prises lors du $\mathrm{Xv}^{\mathrm{e}}$ Congrès en 1997 qui fondamentalement équivalent à une privatisation partielle ou totale des entreprises publiques), la transformation en sociétés de capitaux [de très nombreuses entreprises] est loin d'être anodine ${ }^{49}$.

Et l'auteur d'identifier l'objectif chinois au russe - l'effort organisationnel en plus pour celui-là, en moins pour celui-ci. La loi sur les sociétés de 1993 offre, outre la faculté de constituer des sociétés à capitaux entièrement publics (Titre 2, chapitre 3 ), la possibilité de commercialiser des titres de participation au capital d'entreprises d'État, mais n'impose aucunement que ces entreprises

entreprises d'État, nommée Bureaucrats in Business, se révèlent encore trop lacunaires pour étayer les a priori du libéralisme à l'encontre des entreprises d'État, cf. Luke Haggarty et Mary Shelley, «A new data base on state-owned entreprise ", The World Bank Economic Review, 11 (5), 1998, p. 491-506.

49 Tan Anjie [Tam On Kit], Zhongguo qiye xin tizhi : dudao jizhi yu qiye xiandai hua, Hong Kong, 1998, p. 33. 
- devenues des sociétés à capitaux publics - soient ipso facto tenues pour des entreprises privatisées, ce d'autant moins que certaines participations (celles de l'État, des travailleurs...) ne peuvent être ni cédées, ni négociées librement. Cette opération est si éloignée d'une privatisation que certains ont pu considérer que l'on pouvait opérer une distinction entre le droit de propriété et le droit des actionnaires (guquan). L'intérêt d'une telle prémisse est qu'elle justifie le maintien de zhanyou comme attribut du droit de propriété : un actionnaire ne possède ni ne détient l'entreprise dont il a acheté des titres; en revanche celui qui a reçu le droit de l'administrer, possède cette entreprise d'État du fait même des attributs du droit d'administration (jingyingquan), lequel manifeste le droit de propriété de l'État. Cette construction intellectuelle présentait néanmoins une lacune, elle ne s'appliquait que difficilement hors du secteur étatique. On ne s'étonnera donc pas de sa désuétude ${ }^{50}$.

La question de la " privatisation » a été l'objet d'un débat qui a culminé avec l'« affaire Li Youwei " qui semble s'être close avec la tenue du Xve Congrès en septembre 1997. L'histoire commence le 20 novembre 1996 quand le secrétaire du Parti et ancien maire de Shenzhen ${ }^{51}$, Li Youwei, présente devant l'École centrale du parti ses Réflexions à propos de quelques questions concernant le système de propriété. Le texte enflamme l'aile gauche du Parti - i.e. l'aile intégriste - qui fustige les thèses de Li Youwei dans une campagne d'une violence en partie oubliée depuis la Révolution culturelle. Les attaques sont rédigées et publiées sous la forme de samizdat ${ }^{52}$ dès le début de l'année 1998. Elles ne s'arrêtent qu'en septembre de cette même année, une fois Li Youwei réélu membre suppléant du Comité central et l'orthodoxie de ses thèses confirmée par le discours de Jiang Zemin ${ }^{53}$.

50 Cette conception est toujours présente in Shi Shaoxia, Gongsi fa, Jilin, 1996, p. 24.

51 Remplacé par Li Zibin dans cette dernière fonction le 24 mai 1995.

52 Par ce terme russe, désignant des publications diffusées clandestinement, je traduis l'expression chinoise wan yan shu - mot à mot « livre de dix mille paroles».

53 Pour un compte rendu complet, consulter Ma Licheng et Ling Zhijun, Jiaofeng : dangdai Zhongguo sanci sixiang jiefang shilu, Pékin, 1998, p. 351-366. Malgré ses aspects frondeurs et affranchis, cet ouvrage reste « politiquement correct». 


\section{Thierry Pairault}

Les Réflexions utilisent très adroitement la théorie marxiste du développement des forces productives pour introduire et justifier les positions de Li Youwei qui, dès lors, peut sans crainte - du moins le croyait-il expliciter ce que signifie "privatiser" (siyouhua). Il proclame que par "privatisation », il faut entendre céder - gracieusement ou non - des actifs d'État sous forme de titres à des employés ou à des individus ; il indique en outre qu'une telle procédure semble aujourd'hui précipitée et inadaptée. En revanche, il affirme que transformer les entreprises d'État en sociétés par actions (gufenhua), laisser se développer un secteur privé (i.e. hors la vente d'entreprises d'État) et autoriser la commercialisation d'actifs d'État ne doivent nullement être considérés comme des privatisations. Il ajoute enfin que « ce serait une erreur historique » de ne pas recourir à ces trois procédés puisqu'ils correspondent au niveau actuel de développement des forces productives. Le texte de Li Youwei peut être aujourd'hui consulté sur un site Internet du gouvernement chinois où le bref commentaire l'accompagnant juge sa lecture " rafraîchissante " ${ }^{54}$. On ne saurait exprimer plus clairement que les idées de ce principal responsable de Shenzhen ne choquent en rien les actuels dirigeants chinois!

\section{Réviser sa théorie pour sauver sa doctrine}

Bref, les dirigeants chinois ont défini une stratégie réformatrice qui consiste, pour reprendre la savoureuse expression de René Passet, à « réviser sa théorie pour sauver sa doctrine " ${ }^{55}$; c'est ce dont témoignent à l'envi les propos tenus par Jiang Zemin dans un discours prononcé le 28 juin 1999 pour le $78^{\mathrm{e}}$ anniversaire de la fondation du $\mathrm{PCC}^{56}$ :

54 L'adresse est la suivante : http://www.cei.gov.cn/sicnet/a/m/a/ma981211.htm. Dans l'adresse, «CEI» est le sigle de «China Economic Information » qui dépend de la Commission d'État au Plan. Le texte était accessible dès le 21 janvier 1998.

55 René Passet, L'économique et le vivant, Paris, Economica, 1996, p. 36.

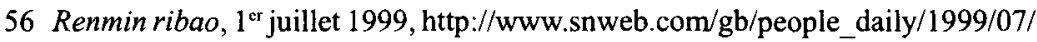
01/a0701001.htm. 
Lénine a dit qu'« une classe qui n'observerait pas les problèmes d'un point de vue politique, perdrait tout contrôle " [...] Le XVe Congrès du Parti a décidé que, pour développer les forces productives, on explorerait diverses formes de propriété publique (gongyou zhi shixian xingshi), en particulier l'actionnariat ; c'est pourquoi certains à l'étranger ont conclu que la Chine se mettait à privatiser. Ici aussi certains camarades ont commis la même erreur, laquelle a conduit à des déviations dans certaines régions. [...] Les actifs d'État s'élèvent à huit mille milliards de yuan; ils sont notre patrimoine commun ; ils sont la base principale de notre économie socialiste. Si on ne restreignait pas l'appropriation individuelle d'actions, le risque serait grand qu'elles soient accaparées par une minorité et que l'on perde tout contrôle sur nos actifs d'État, que les fondements de notre système socialiste soient sapés. [...] C'est pourquoi j'insiste pour que nos cadres dirigeants, en particulier ceux de grade élevé, ne se contentent pas de voir les problèmes du seul point de vue de l'économie, mais observent et résolvent les problèmes dans une perspective résolument politique.

Et la Décision du PCC sur quelques problèmes concernant le développement et la réforme des entreprises d'État (22 septembre 1999) ${ }^{57}$ d'insister sur les conditions politiques et de proposer des aménagements financiers, juridiques et sociaux nécessaires à la transformation des entreprises d'État en sociétés de capitaux qui puissent en même temps conserver à la Chine un patrimoine public et préserver les principes établis.

57 Renmin ribao, 27 septembre 1999, http://www.snweb.com/gb/people_daily/1999/ 09/27/a0927001.htm. 


\section{Bibliographie}

Alchian Armen A., «Property Rights », The New Palgrave : A Dictionary of Economics, London, Macmillan,1987, p. 1031-1034.

Bettelheim Charles, La transition vers l'économie socialiste, Paris, François Maspéro, 1977, 268 p. («Économie et socialisme ", n 9).

Chambre Henri, L'évolution du marxisme soviétique : théorie économique et droit, Paris, Seuil, 1974, 476 p. ("Esprit - Frontière ouverte »).

Coase Ronald H. "The Nature of the Firm », Economica, vol. IV, November 1937,

p. 386-405 (traduction française : "La nature de la firme », Revue Française d'Économie, vol. 11, n 1, hiver 1987, p. 133-163).

Coase Ronald H., «The Problem of Social Cost », The Journal of Law and Economics, vol. III, October 1960, p. 1-44 (Traduction française : « Le problème du coût social », Revue Française d'Économie, vol. VII, nº 4, automne 1992, p. 153193).

Demsetz Harold, « Toward a Theory of Property Rights ", The American Economic Review, vol. LVII, ${ }^{\circ} 2$, May 1967, (Papers and Proceeding of the Seventy-ninth Annual Meeting of the American Economic Association), p. 347-359.

Dong Fureng, « Guanyu woguo shehui zhuyi suoyou zhi xingshi wenti (La question des modes d'appropriation socialiste en Chine), Jingji yanjiu, 1979, n 1 , p. $21-$ 28.

Faxue cidian (Dictionnaire de science juridique), Shanghai, Shanghai cishu chubanshe, $1980,742+10 \mathrm{p}$.

Haggarty Luke et Shelley Mary, "A new data base on state-owned entreprise », The World Bank Economic Review, 11(5), 1998, p. 491-506.

Jaurès Jean, « De la propriété socialiste », in Études socialistes..., Paris, P. Ollendorff, 1902 (document consulté : numérisation de la BNF de l'édition de Paris: INALF, 1961 [Franxtext, L 499]).

Li Xiangru (éd.), Taiwan wuquan fa (Les droits réels à Taiwan), Beijing, Zhongguo guangbo dianshi chubanshe, 1993, $221 \mathrm{p}$.

Li Xinxin, «Liaoning, Fujian liang zheng suoyou da zhong xing qiye mianlin de kunjing ji qi yuanyin fenxi » (Des difficultés rencontrées par les grandes et moyennes entreprises des provinces du Liaoning et du Fujian et analyse de leurs causes), Jingii yanjiu ziliao (Documents de la revue Études économiques), 1997(4), p. 15-21.

Li Yining, « Gaige jiben silou » (Réflexions pour la réforme), Beijing ribao, 19 mai 1986, p. 3. 


\section{Droit de propriété et réforme du secteur d'État}

Li Yining, "Woguo suoyou zhi gaige de shexiang " (Propositions pour la réforme des modes d'appropriation en Chine), Renmin ribao, 26 septembre 1986, p. 5.

Liang Shuwen et al. (éd.), Minfa tongze ji peitao guiding xin shi xin jie (Nouvelles explications des Principes généraux du droit civil et des textes d'application), Beijing, Renmin fayuan chubanshe, 1996, $1146 \mathrm{p}$.

Lin Yifu [Justin Lin] et al., Chongfen xinxi yu guoyou qiye gaige (=Sufficient Information and State-Owned Entreprise Reform), Shanghai, Shanghai renmin chubanshe et Shanghai sanlian shudian, 1997, $178 \mathrm{p}$.

Ma Licheng et Ling Zhijun, Jiaofeng : dangdai Zhongguo sanci sixiang jiefang shilu (Affrontements : compte-rendu de trois combats pour l'émancipation intellectuelle en Chine aujourd'hui) Beijing, Jinri chubanshe, 1998, $426 \mathrm{p}$.

Ma Zhuotan et al., "Lun touzi yu qiye de guoyou zichan shang de caichan quan » (De l'investissement dans l'entreprise et des droits patrimoniaux sur les actifs d'État), Faxue zazhi (La revue du droit), 1996 (6), p. 8-9.

Maréchal Jean-Paul, Éléments d'analyse économique de la firme, Rennes, Presses Universitaires de Rennes, 1999, $221 \mathrm{p}$.

Marx Karl, Contribution à la critique de l'économie politique, Paris, Éditions sociales, $1977,309 \mathrm{p}$.

Ota Ban, Faxue tonglun (De la science juridique), Shanghai, Shangwu yinshuguan, 1907 [traduit du japonais par Liu Chongyou].

Passet René, L'économique et le vivant, Paris, Economica, 1996 (1 ${ }^{\mathrm{rc}}$ édition, Payot, 1979).

Pigou Arthur C., The Economics of Welfare, London, Macmillan, 1920, 976 p.

Shi Shangkuan, Wuquan fa lun (Des droits réels), Taipei, Shi Wu Zhongfang et Shi Guanghua, Jinhua jie, 32 xiang, 2 long, 4F, 1957.

Shi Shaoxia, Gongsi fa (xiuding ban) (La loi sur les sociétés - édition revue et corrigée), Jilin, Jilin renmin chubanshe, 1996, $380 \mathrm{p}$.

Sun Yefang xuanji (Euvres choisies de Sun Yefang), Taiyuan, Shanxi renmin chubanshe, 1984, $874 \mathrm{p}$.

Starr Paul, « The meaning of privatization », Yale Law and Policy Review, 1988 (6), p. 6-41.

Tan Anjie [Tam On Kit], Zhongguo qiye xin tizhi : dudao jizhi yu qiye xiandai hua (Le nouveau système d'entreprises en Chine : contrôle et modernisation des entreprises), Hong Kong, Shangwu yinshuguan, 1998, 186 p.

Tong Ruo, Zhonghua faxue da cidian : minfaxue juan (Grand dictionnaire chinois de science juridique : droit civil), Beijing, Zhongguo jiancha chubanshe, 1995.

Wang Jiafu (éd.), Zhongguo minfa xue : minfa zhaiquan (Droit civil chinois : les obligations), Beijing, Falü chubanshe, 1994, 898 p. 


\section{Thierry Pairault}

Wang Kaiguo (éd.), Zhongguo qiye chanquan jiaoyi shiwu wenda (Questions et réponses sur le transfert d'actifs des entreprises chinoises), Beijing, Zhongxin chubanshe, $1995,635 \mathrm{p}$.

Wang Liming, "Guojia suoyouquan de falü tezheng yanjiu » (Caractéristiques légales du droit de propriété étatique), Falü kexue (Science juridique), 1990, nº 6, p. 29 35.

Wang Liming, «Suoyouquan minfa baohu de ruogan wenti » (À propos de la préservation du droit de propriété en droit civil), Falü xuexi yu yanjiu (Études et recherches en science du droit), 1990, $n^{\circ} 4$, p. 57-62.

Wang Liming, «Lun guojia suoyouquan de minfa baohu » (De la protection de la propriété étatique en droit civil), Zhongguo faxue (Science juridique en Chine), $1991, n^{\circ} 2$, p. $85-90$.

Wang Liming, Gaige kaifang zhong de minfa yinan wenti (Problèmes ardus de droit civil affectant l'ouverture et la réforme), Changchun, Jilin chubanshe, 1992.

Wang Qufei, Minfa wu quan lun (Des droits réels), Shanghai, Shanghai faxue bianyishe, 1930, $326 \mathrm{p}$.

Wang Xiaolong et al., " Guoyou qiye chanquan yu xiandai qiye zhidu » (Les droits patrimoniaux des entreprises d'État et le système des entreprises modernes), Xiandai qiye baodao (=Modern Entreprise Herald), $\mathrm{n}^{\circ} 167,1997$ (5), p. 58-60.

Wang Zuotang et al., Beijing daxue shiyong jiaocai : minfa jiaocheng (Matériaux pédagogiques expérimentaux : manuel de droit civil), Beijing, Beijing daxue chubanshe, 1982.

Yan Qifa, "Chanquan wenti yanjiu zongshu » (Résumé des recherches sur la question des droits patrimoniaux), Zhongguo qiye guanli nianjian - 1996 (Annuaire de la gestion des entreprises en Chine - 1996) Beijing, Qiye guanli chubanshe, 1996, p. 398-401.

Yang Lixin, « Woguo ta wu quan zhidu de chongxin gouzao » (La reconstruction du système des droits réels sur la chose d'autrui en Chine), Zhongguo shehui kexue (Sciences sociales en Chine), 1995, n³, p. 75-93.

Ye Xiaoxin (éd.), Zhongguo minfa shi (Histoire du droit civil chinois), Shanghai, Shanghai renmin chubanshe, 1993, $654 \mathrm{p}$.

Zhang Zhiguang et al., "Guanyu faren caichan quan de jige wenti" (Quelques questions sur le droit de propriété des personnes morales), Dongbei shida xuebao (Journal de l'École normale du Dongbei), 1995, nº 5, p. 31-37 et p. 41. 


\section{Caractères chinois}

caichan quan 財產權

caichan suoyouquan 財產所有權

Caichan suoyouquan he yu caichan suoyouquan youguan de caichan quan 財產所有權和與財産所有權有關的財產權

caiquan qingxi 財權清晰

chanquan 產權

chanquan guanxi 產權關係

chanquan xuepai 產權學派

chiyou 持有

chufen 處分

danbao wu quan 擔保物權

dian quan 典權

dingxian wu quan 定限物權

duiyu wu you shishi shang guanling zhi li zhe, wei zhanyou ren

對于物有事實上管領之力者, 爲佔有人

falü shang zhanyou 法律上佔有

fei gongyou 非公有

gongmin 公民

gongmin geren caichan suoyouquan 公民個人財產所有權

gongsihua 公司化

gongying qiye 公營企業

gongyou 公有

gongyou minying qiye 公有民營企業

gongyou qiye 公有企業

gongyou zhi shixian xingshi 公有制寅現形式

gufenhua 股份化

guquan 股權

Guanyu suoyou zhi ruogan wenti de sikao

關於所有制若干問題的思考

guojia caichan shensheng bu ke qinfan 國家財產神聖不可侵犯

guoyou qiye 國有企業

hong maozi 紅帽子

jingyingquan 經營權

leisi wu quan 類似物權

Li Youwei 厲有営

“ mai qiye" zhi feng “賣企業”之風

Minfa tongze 民法通則 


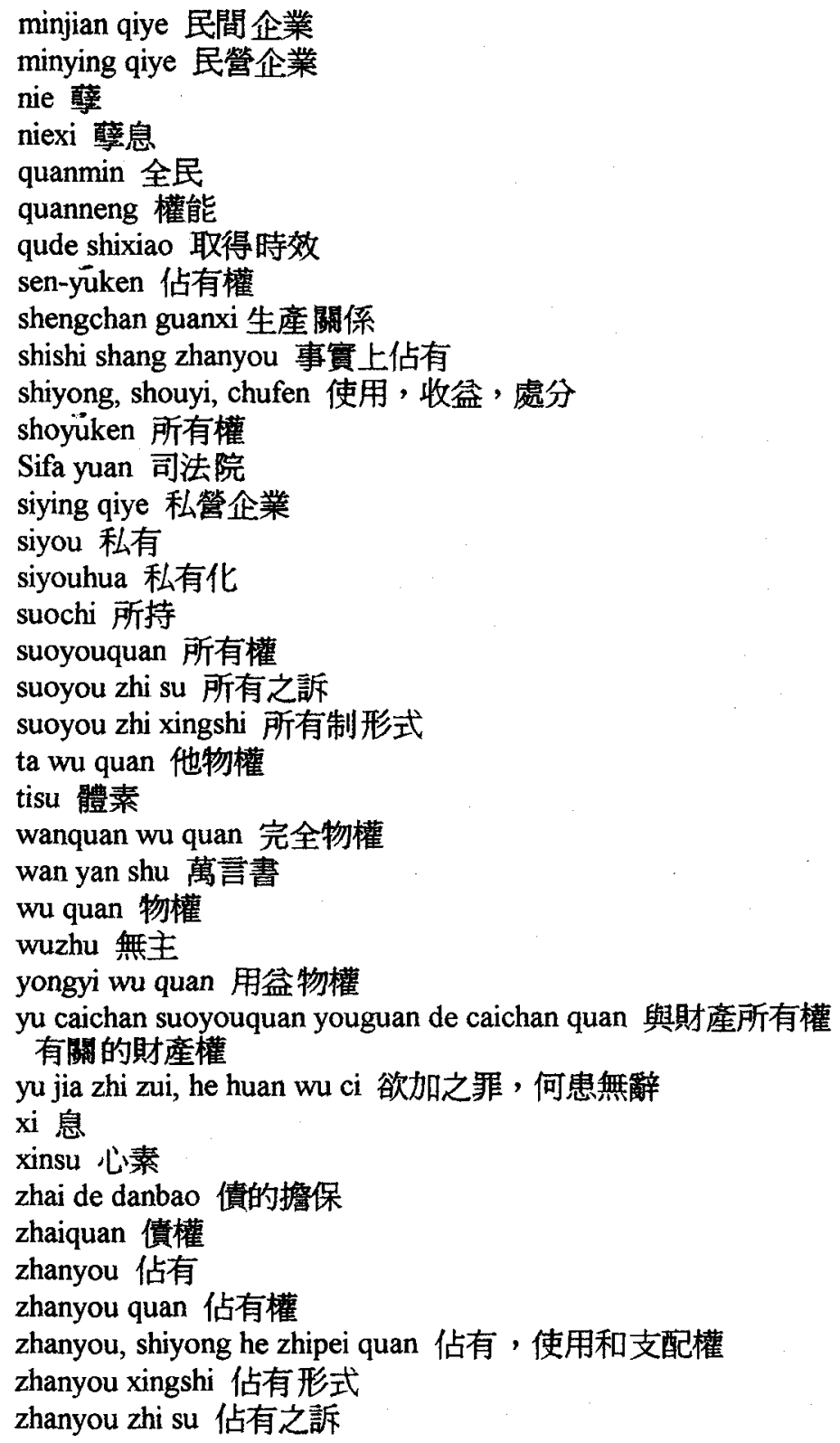


Droit de propriété et réforme du secteur d'État

zhipei 支配

zhi quan 質權

zi 掱

zichan quan 資產權

ziran ren 自然人

zi wu quan 自物權

zixi 紊息 


\title{
Thierry Pairault
}

\section{Résumé}

Thierry PaIRAuLt : Droit de propriété et réforme du secteur d'État

La question des droits patrimoniaux relatifs à la propriété et du droit de propriété en Chine dans le contexte de la réforme du secteur d'État est l'objet de cette étude. Afin d'assumer l'héritage du système d'appropriation, les dirigeants chinois ont été régulièrement confrontés à la nécessité de clarifier ces droits patrimoniaux. Une telle clarification est particulièrement cruciale pour les entreprises d'État dont le statut est étroitement lié à la légitimité d'un " socialisme à la chinoise ». Aux termes de la Constitution, « l'État maintient la propriété publique comme élément principal dans un système économique où plusieurs modes d'appropriation se développent ensemble ". Une telle diversité d'intérêts divergents suppose une mutation des droits patrimoniaux et des obligations du secteur d'État. La restructuration des entreprises d'État doit-elle être interprétée comme leur privatisation ? Dans quelle mesure la clarification des droits patrimoniaux conduit-elle inévitablement à une redistribution du droit de propriété ?

\begin{abstract}
Thierry PaIRault: Property Rights and Reform of the State sector

The issue of property rights and ownership relatively to the reform of the state sector in China is the main concern of this article. In the attempt to deal with the legacy of the ownership system, the Chinese leadership has repeatedly been contemplating clarifying the property rights. The need of such a clarification is especially crucial to the stated-owned enteprises as their status is closely linked to the legitimacy of "building socialism with Chinese characteristics". According to the Constitution, "the State adheres to the basic economic system with the public ownership remaining dominant and diverse sectors of the economy developing side by side". Such pluralism of competing interests implies the transformation of property rights and obligations of the State sector. Should restructuring stated-owned enterprises be understood as their privatisation? To which extent clarifying property rights should inevitably result in the reallocation of ownership right?
\end{abstract}

